\title{
Natural Selection of Independently Originated Life Clades
}

\author{
Margarida Hermida \\ University of Bristol, Department of Philosophy, Cotham House, Bristol, UK \\ Email: margarida.hermida@bristol.ac.uk
}

(Received 08 November 2020; revised 06 April 2021; accepted 24 June 2021; first published online 12 January 2022)

\begin{abstract}
Life on Earth descends from a common ancestor. However, it is likely that there are other instances of life in the universe. If so, each abiogenesis event will have given rise to an independently originated life clade (IOLC), of which Earth-life is an example. In this paper, I argue that the set of all IOLCs in the universe forms a Darwinian population subject to natural selection, with more widely dispersed IOLCs being less likely to face extinction. As a result, we should expect that, over time, more planets will become inhabited by fewer, more successful IOLCs.
\end{abstract}

\section{Introduction}

The study of life in the universe is one of the most intellectually exciting human pursuits, and the discovery of extraterrestrial life, or even evidence of extinct life unrelated to Earth-life, would be, without a doubt, the most important discovery of all time. Despite the extraordinary diversity of life on Earth, with a vast array of organisms using different kinds of metabolism and energy sources (e.g., phototrophy, chemotrophy, heterotrophy), all this diversity was generated by evolution from a single origin. For that reason, life on Earth effectively constitutes a single sample of life, a problem known as $\mathrm{N}=1$.

Due to this limitation, we have been unable to develop a theoretical framework for the understanding of life that goes beyond simply listing the features of Earth life we find particularly important (Cleland and Chyba 2002). For this reason, many authors have assumed a pessimistic view of the possibility of defining life (e.g., Cleland 2012, 2019; Machery 2012), with some authors going so far as to consider the question to be incoherent in the first place (Mariscal and Doolittle 2018). Other authors, however, point to the usefulness of pragmatic, operational, or diagnostic definitions of life for astrobiology research (Knuuttila and Loettgers 2017; Bich and Green 2018). While there is no doubt that a second example of life would be a huge step towards our understanding of life in the universe and the development of an adequate Theory

(c) The Author(s), 2022, Published by Cambridge University Press on behalf of the Philosophy of Science Association. This is an Open Access article, distributed under the terms of the Creative Commons Attribution licence (http:// creativecommons.org/licenses/by/4.0/), which permits unrestricted re-use, distribution and reproduction, provided the original article is properly cited. 
of Life (Cleland 2019), it would not magically solve the problem, as it would only raise our sample from one to two. It would not allow us to claim that the features shared by both examples of life were universal.

Nonetheless, it is possible to contribute to the programme of universal biology, and even make some progress towards a future Theory of Life, in the absence of a second sample of independently originated life (Mariscal and Fleming 2018; Segura et al. 2020). The purpose of universal biology is not just to identify which features of life on Earth are contingent and which are universal, but also to clarify the different concepts associated with the phenomenon of life (Hermida 2016; Segura et al. 2020) and, I suggest, to investigate the structure of life in the universe from a distinctly biological perspective. This paper aims to contribute to that project by advancing a hypothesis concerning life in the universe.

Since life on Earth descends from a common ancestor, it constitutes a large monophyletic clade. If other instances of life exist in the universe, each abiogenesis event will presumably give rise to an independent clade. In this paper, I argue that the set of independently originated life clades in the universe constitutes a Darwinian population subject to natural selection. The proposed theory gives an account of the structure of life across the universe in terms of specifically biological categories and extends the domain of application of natural selection. This is important, since much theoretical work concerning the nature of life is based on concepts and categories borrowed from other sciences, e.g., thermodynamics (Schrödinger 1992), chemistry (Pross 2012), or information (Korzeniewski 2001). This work aims to bring all life in the universe within the scope of biology, which we have good reason to believe is a truly universal science, despite the dearth of study samples so far.

The structure of the paper is as follows. In section 2, I clarify the concepts discussed, including "life," "Earth-life," "IOLC," and "biosphere." In section 3, I introduce the concept of IOLC selection as a particular kind of clade selection and explain how it escapes much of the criticism levelled at traditional cases of clade selection. In section 4, I argue that expansion to other planets constitutes a form of quasireproduction and discuss the ways in which it contributes to IOLC fitness. Sections 5 and 6 focus specifically on IOLC selection and competition between IOLCs, respectively. Section 7 discusses possible implications of the theory for the distribution of life in the universe over time. Finally, in section 8, I explain how the theory proposed in this paper contributes to clarifying the place of life as a general concept, and of Earth-life as an individual, in scientific theory.

\section{Earth-life as an individual vs. life as a natural kind}

To begin with, it is important to clarify a few concepts. I understand life to refer to the process that is instantiated by living systems, such as organisms. However, while the life process is instantiated in individual organisms, these can only evolve in the context of large historical-collective individuals (Ruiz-Mirazo et al. 2004), or life-individuals, of which life on Earth is an example (Hermida 2016). I propose to further specify this kind of life-individual as the maximal clade that includes all the descendant organisms from a particular abiogenesis, or origin of life, event, and I will refer to this kind of maximal clade as an independently originated life clade (IOLC). 
I will not attempt here to adjudicate between different definitions of life, or defend my own preferred definition, as that would fall outside the scope of this paper. In fact, I find it very plausible that we are not, at present, in a condition to formulate a complete theory of life (Cleland 2019), and for that reason, all proposed definitions may be inadequate. However, the aim of this paper is somewhat orthogonal to the debate concerning the definition of life.

For present purposes, I will assume that life is a natural kind, albeit one of which we are at present unable to give a satisfactory definition, but whose nature will be better elucidated in the context of a future Theory of Life. Nevertheless, I think there are good reasons why we are justified in thinking that life is indeed a natural kind. The main reason is that it is overwhelmingly likely that there are more instances of life in the Universe, and, moreover, this is true regardless of which definition of life is employed. For instance, even if we were to restrict the scope of "life" to "life as we know it"- that is, carbon based, using liquid water as a medium -it would still be overwhelmingly likely that Earth-life is not the only instance of this phenomenon. In fact, given the ample abundance of carbon, water, and potential energy sources in the universe, the spontaneous occurrence of molecules that serve as building blocks for Earth-life, ${ }^{1}$ and how quickly it originated on Earth as soon as the heavy bombardment ceased (Lineweaver and Davies 2002), it is extremely unlikely that Earth-life should be the only instance. Furthermore, given the vastness of the universe, many instances of life are likely to exist even if abiogenesis is extremely rare (Schulze-Makuch and Irwin 2006).

In addition, although it is certainly important to be open-minded regarding the possibilities for extraterrestrial life (otherwise, unexpected kinds of life might go undetected), we should also avoid the contrary tendency of assuming that alien life necessarily must be strange or anomalous. We know that life based on proteins, lipids, DNA, etc, is possible, and we know that both carbon and liquid water are particularly amenable to complex biochemistry. And although we might currently be unable to converge on a satisfactory definition of life, it is also not the case that we do not know anything about life and some of its constraints. In other words, while alien life might certainly prove strange and problematic, we should not expect all instances of alien life to be problem cases.

Even bracketing most assumptions regarding the nature of life, I take it that it is relatively uncontroversial that life cannot emerge as a single isolated organism. While Swampman might be metaphysically possible, in the actual world, it is highly unlikely that a living organism would emerge on its own and not as part of a population within an evolving lineage. Nevertheless, if a particular abiogenesis event were to give rise to a single isolated organism with no reproductive capacity, the theory of IOLC selection as proposed in this paper would still apply - the solitary organism would constitute a very uninteresting IOLC composed exclusively of itself.

In the case of life on Earth, the IOLC is composed of FUCA (the first universal common ancestor) and all its descendants, both living and extinct. I refer to this particular individual IOLC as Earth-life. An important question that might arise is

\footnotetext{
${ }^{1}$ For instance, amino acids, nucleobases, and even sugars including ribose, a component of RNA, have been discovered in meteorites (Furukawa et al. 2019).
} 
whether viruses and other acellular entities should be included in this clade. ${ }^{2}$ For instance, the account of life on Earth as an individual in Hermida (2016) implicitly excludes these by considering continuity at the cellular level as an important feature of the individuality of this historical-collective entity. However, the theory I propose in this paper is compatible with either inclusion or exclusion of acellular entities.

Life on Earth also forms a biosphere, which is associated with a particular planetEarth - but is not coextensive with the Earth-life IOLC, since it is possible for Earthlife to expand to other planets, but it is not possible for the Earth's biosphere to do so, since a biosphere is, by definition, associated with a given planet.

Some authors refer to Earth-life as "Life" (Mariscal and Doolittle 2018; Doolittle 2019; Lenton et al. 2020). This terminology is somewhat misleading, however, since it seems to imply that Earth-life is the only life that exists in the Universe, something which, although possible, is highly unlikely. Therefore, in this paper I will use life as the generic term, and Earth-life as a name for the particular IOLC that originated on Earth. Furthermore, while these authors consider "Life" to include all and only the descendants of LUCA (the last universal common ancestor), I see no reason to exclude the ancestors of LUCA, since my aim is to identity the maximal clade. I consider an IOLC to include all the descendants of an origin-of-life event, extant or not.

\section{Natural selection, clade selection, and IOLC selection}

Evolution by natural selection is expected to be true for any life phenomena, anywhere in the universe, regardless of the specific details of alien life forms, much as physical and chemical laws are reasonably expected to be true across the universe (Dawkins 1983; Sterelny and Griffiths 1999; Levin et al. 2019).

Although natural selection may act primarily upon individual organisms, other biological levels of organization can also be targets of selection including, among others, cell lineages, symbiotic partnerships, species, and possibly higher taxonomic levels. In fact, natural selection can act upon any given population of things, provided they satisfy the following criteria (Lewontin 1970; Okasha 2006; Godfrey-Smith 2007):

(1) the entities that make up the population should exhibit variation among themselves;

(2) these differences should correlate with differences in fitness;

(3) the differences are heritable, i.e., they tend to be transmitted from parents to offspring.

Any population of things that satisfies these criteria constitutes a Darwinian population, which can be subject to natural selection (Godfrey-Smith 2009). Here, I propose that the set of all independently originated life clades in the universe satisfies these conditions and constitutes a Darwinian population subject to natural selection. Let us examine how closely IOLCs satisfy each criterion.

Condition (1) is very easily acceptable. While there is ample discussion concerning how different independently originated instances of life are likely to be, few would

\footnotetext{
${ }^{2}$ I thank an anonymous reviewer for this point.
} 
think it plausible that all life in the universe should not vary in important respects. At the very least, there are likely to be differences in biochemistry, different genetic codes, and so on. Furthermore, the particular evolutionary history of each IOLC will necessarily involve a high degree of contingency, which will constrain its further evolutionary possibilities. It is simply implausible that a population of IOLCs should not exhibit variation.

If there is variation, then it is very likely that some features should be more favorable than others. For instance, it might be the case that some biochemical features are more versatile than others and allow for higher evolvability. Other favorable features might include wide distribution range within its home planet and capacity for interplanetary expansion, since both will reduce the probability of extinction of the IOLC (much as large population size and wide distribution range are features that reduce a species' extinction risk). Thus, we can conclude that condition (2) also applies.

Condition (3) implies that the entities in question undergo some form of reproduction. Okasha $(2006,14)$ argues that it is essential to the notion of reproduction that parental and offspring entities belong to the same level in the biological hierarchy, and that offspring entities can outlive parental ones (Okasha 2006, 213). In the case of species, while they might not literally reproduce, speciation plays the role for species that reproduction does for organisms, resulting in the production of at least one new entity of the same kind, which can outlive the parental entity.

IOLC selection, as proposed in this work, is a form of clade selection. The possibility of clade selection has been proposed, with some authors arguing that cladogenesis is the equivalent of reproduction at clade level—cladogenesis would be to clades what speciation is to species (Haber and Hamilton 2005). But there is an important difference: when the process of speciation is complete, at least one new species is not part of the former species, whereas when a clade divides, all of its descendants are still part of the original clade; that is what it means to be a monophyletic clade. This is why Okasha $(2003 ; 2006,213-14)$ considers clade selection to be conceptually incoherent: sub-clades cannot outlive the parent clade; therefore, it is meaningless to talk about clade fitness.

Some clade selection theorists counter that clade fitness can be redefined as higher survival or differential persistence (Doolittle 2017). In fact, it is possible to defend a more encompassing notion of fitness that includes not only multiplication, but also expansion and persistence (Van Valen 1976). Under this wider conception of fitness, a plant that covers a whole field by sending out clonal runners is fitter than one that consists of a single shoot, and an organism that lives for thousands of years is fitter than a short-lived one (Simpson 2011). This makes intuitive sense, since, in both cases, the more dispersed or longer-lived organism is, as a matter of fact, much more resistant to destruction. Nevertheless, other authors counter that differential persistence is not enough to ground a significant Darwinian process in the absence of reproduction (Godfrey-Smith 2009, 152). Reproduction might, however, still not be strictly necessary for natural selection to occur; a reproductive-like process may be enough, as long as this reproductive-like process permits multiplication (Godfrey-Smith 2009, 85, 104).

IOLC selection is a form of clade selection, but it is very unlike ordinary clade selection in at least two ways. First, the clades in question are genuinely independent, not phylogenetically related. Second, cladogenesis is not the form of "reproduction" 
proposed, which is arguably the most problematic feature of clade selection. Instead, it is proposed that interplanetary expansion constitutes a form of quasi-reproduction. Furthermore, interplanetary expansion extends the distribution range of the expanding IOLCs in a way that significantly reduces their extinction risk, thus promoting their long-term survival, which constitutes higher fitness, according to a broad definition of fitness.

Clade selection is based on differences in fitness among clades (Williams 1992, 25), which translate into different probabilities of extinction. ${ }^{3}$ These differences in fitness might come about in various ways. For IOLCs, differences in fitness might result from what we may consider to be essential, or at least "frozen accidental" properties of an IOLC, such as its biochemical basis, established in the abiogenesis event; or it might result from the combined fitnesses of their component biosphere(s), such as their successful establishment in interaction with their particular planets and the biodiversity achieved over its evolutionary history; or it might result from emergent properties of the IOLC itself, such as its distribution range.

\section{Interplanetary expansion as quasi-reproduction and as range expansion}

Before moving on to IOLC selection proper (sections 5 and 6), we will look at how interplanetary expansion and the successful establishment of additional biospheres in non-native planets contributes to IOLC fitness by functioning as a quasireproductive process that increases the distribution range of the IOLC and its intra-clade diversity. Both features contribute to the IOLC's longevity over time by lowering its probability of extinction. Although IOLCs, like any clade, cannot reproduce per se, interplanetary expansion can function as a quasi-reproductive process, since a new biosphere established on a different planet can become independent of the original one.

To see how expansion of an IOLC to another planet could be seen as a reproductivelike process, suppose that humans were to colonize Mars. To colonize another planet, humans would need to carry plants or other photosynthetic organisms to produce oxygen, food to eat, and so on. In fact, colonists would probably need to carry several organisms to fulfill various needs, not to mention the microbial communities within their own bodies. In this sense, we would effectively be carrying a "propagule" of Earth-life to another planet.

Although this transfer of a part of Earth-life from one planet to another would not constitute true reproduction, since it would not result in an independent clade, which is impossible by definition, it would nonetheless result in the production of an entity-a new biosphere-which, despite belonging to the same clade, could go on to have an independent existence in the new planet. This new entity would most likely follow a different evolutionary path and could even continue to exist after the demise of the parental entity, if life on Earth were to become extinct after life had already become established on Mars. Thus, the "quasi-reproduction" of Earth life would satisfy all of Okasha's $(2003,2006)$ requirements for the occurrence of

\footnotetext{
${ }^{3}$ Williams explains these differences in fitness in terms of differences in the ratio of cladogenesis and extinction, but, in this paper, I disregard cladogenesis altogether.
} 
reproduction: the generation of a new entity at the same level of biological organization and its independence from the parental entity.

Furthermore, the expansion of life from one planet to another would involve the transfer of only a relatively small propagule, as it would not be possible to transport all of the original biota's component parts. In this context, a propagule is any organism or group of organisms that can disperse the IOLC through interplanetary travel. This would in effect function as a bottleneck, which is a common feature of reproductive events and creates the conditions for a strong "founder effect," and differentiates the new entity even more from the parent entity (Godfrey-Smith $2009,105)$. Although at first a small subset of the original planet's life, the offspring aggregate would soon start to experience a different set of evolutionary constraints on the new planet. In the hypothetical example of Earth-life expansion to Mars, different prevailing conditions on that planet, including differences in temperature, lower gravity, red-heavy light spectrum, different atmosphere, et cetera, would begin to exert selective pressures on the terrestrial organisms in completely different directions as compared to Earth, with evolution on the new planet likely to follow entirely different paths.

The independent existence of the new biosphere would depend on the number of organisms and other materials exchanged between the two planets. Thus, if initially there were frequent exchanges of material between the two planets, we might not be able to speak of true independent existence, but the two planets would be interconnected in much the same way that parent and offspring plants can be connected through the root system, exchanging nutrients and so on, but this connection can be severed, making the offspring plant fully independent. In any case, the potential for independent existence is what is needed for the offspring to be considered a different entity from the parent. The connection could be severed at any moment, and the two biospheres would continue their independent existence.

The expansion of life from Earth to Mars in our hypothetical example could be described in two ways: as the expansion of the distribution range of a particular IOLC (Earth-life), or as a quasi-reproductive event in which the Earth's biosphere gives rise to an independent biosphere on a non-native planet. The biosphere can be seen as analogous to an asexually reproducing organism, while the IOLC is analogous to the species or lineage the organism belongs to. Thus, we can make a parallel with species selection, where the fecundity and dispersal capacity of individual organisms gives rise to an emergent property of the species-wider distribution range-which confers higher fitness to the species itself. Similarly, IOLCs with wider distribution ranges, especially if spread across different planets, will be fitter than IOLCs with more restricted distribution ranges, especially those restricted to a single planet (see discussion in section 5).

At this point, we consider the possibility that selection might operate on biospheres as well. After all, biospheres are also likely to exhibit variation, with some traits being more favorable than others. However, there are two important aspects to consider. First, what is transported in interplanetary expansion is a small propagule, which, while it certainly carries some of the traits of the IOLC (for instance, its basic biochemistry, or its evolvability), it is unable to carry the properties of the biosphere, which contribute to its longevity in its previous planet; in fact, even if quite a large propagule were carried, this would still not suffice to carry the biosphere's traits with 
it, since those are specific to the particular life/planet interaction. Second, different biospheres are very unlikely to experience similar selective pressures. Instead, each experiences a different set of selective pressures specific to the particular planet. ${ }^{4}$ This makes talking about selection among biospheres somewhat problematic.

On the other hand, the diversity among biospheres that is likely to result from those different selective pressures could itself be another feature of interplanetary expansion that might contribute to emergent IOLC fitness through the increase in intra-clade diversity. For instance, in species selection, it is not only high population size and wide distribution range, but also high intraspecific diversity, that reduce a species probability of extinction.

\section{Natural selection of IOLCs}

If there are multiple IOLCs in the universe, resulting from multiple abiogenesis events, which is likely given the vastness of the universe, and if it is possible for life, at least on occasion, to expand beyond its native planet, then it is natural to assume that some IOLCs will have a higher dispersal capacity than others. This will result in those IOLCs becoming dispersed across multiple habitable planets, whereas IOLCs with lower or no dispersal capacity will be restricted to fewer or to a single planet, namely their native planet. Naturally, more highly dispersed IOLCs will have a lower probability of extinction, since "uniqueness is always more fragile than multiplicity" (Ćirković 2019).

Even in organisms that are well adapted to their environments, numerous threats can lead to extinction. Rapid environmental change and major catastrophic events such as asteroid strikes and widespread volcanism can lead to mass extinctions, but those do not generally completely eliminate all life. Other threats, however, are liable not only to lead to complete destruction of any extant life, but even to render the planet uninhabitable - those include changes in solar diameter and radiation, which happen as part of the normal "life cycle" of main sequence stars, heavy asteroid bombardment, and other astronomical phenomena.

In the absence of interplanetary expansion, a IOLC eventually goes extinct on the planet where it originated, when either (i) a global catastrophic event such as large meteor strike destroys all life on the planet, or (ii) the original planet ceases to be habitable, for instance, due to changes in energy radiating from its star. Scenario (i) may have occurred on Earth, with possible previous life having gone extinct during the late heavy bombardment (Lowe and Byerly 2018), with a later abiogenesis event giving rise to the IOLC Earth-life (but this is controversial; see Abramov and Mojzsis [2009] for a dissenting view). Scenario (ii) is predicted to take place on Earth in about 1 billion years, if runaway climate change does not get us there first. Evidently, if an IOLC is restricted to a single planet, it goes extinct when the planet becomes uninhabitable; this is not necessarily the case for a IOLC that has established functioning biospheres on several planets, since different planets are likely to have different habitability windows.

${ }^{4}$ This is an oversimplification; evidently selective pressures will also vary within each planet and across time. But the point is that each planet will constrain the evolution of its biosphere in different ways (and indeed the biosphere itself will also constrain planetary evolution in particular ways). 
In effect, the population of all IOLCs in the universe constitutes a Darwinian population, in which more expansive IOLCs will be positively selected to the detriment of less- or non-expansive ones. IOLC selection is thus analogous to species selection, as defended, for instance, by Jablonski. According to Jablonski (2008), species selection in the strict sense occurs when emergent traits at the species level, such as geographic range and population size, which cannot be reduced to organism-level traits, confer an advantage on those species in comparison to others, leading to different extinction rates, which determine macroevolutionary patterns across time. A similar situation can happen in the case of IOLCs. If some IOLCs are spread out across several planets, the probability of their extinction is lower than that of IOLCs that are restricted to fewer or a single planet.

Several IOLC traits might increase the probability of successful expansion. To begin with, the successful establishment of a functioning biosphere on the native planet is likely to be essential to the survival of the IOLC until such time as it is able to expand to additional planets. The early development, and long-term maintenance, of harmonious biogeochemical cycles appropriate for the abiotic resources available on the native planet is likely to be crucial for the IOLC's longevity in its native planet (Knoll 2003; Chopra and Lineweaver 2016; Doolittle 2019), which in turn increases the likelihood of expansion.

Chopra and Lineweaver (2016) hypothesize that since the ingredients for life seem to be abundantly available in the universe, and yet life, from what we can observe, seems to be rare, the most likely explanation is that there is a "Gaian bottleneck" that most life does not survive. They assume that planets with conditions for abiogenesis tend to have short habitability windows, unless the planetary biota is able to establish a functioning biosphere that can counteract runaway positive feedbacks, generating a stable environment through the interaction between life and environment, and thereby significantly extending the habitability window of the planet. If their hypothesis is correct, then the capacity of an IOLC to successfully establish a functioning biosphere capable of extending the habitability window of its planet is crucial to extending the IOLC's longevity.

Other IOLC traits that are likely to decrease the probability of extinction during its single-planet phase of existence might be high evolvability, high biodiversity, and wide environmental breadth of its component species, all of which decrease the risk of IOLC extinction during mass extinction events and increase the probability of successful interplanetary expansion, since this probability is likely to increase over time. Equally, high evolvability is essential for the successful establishment of new biospheres once interplanetary transfer has been achieved.

Although in the example above of Earth-life expansion to Mars, interplanetary expansion is achieved through the deliberate technological transport of organisms carried out by humans, there are other possible alternatives. One is that interplanetary expansion might be entirely accidental, the result of the exchange of materials between planets in relatively close proximity coupled with the capacity to survive vacuum exposure and atmosphere re-entry on the part of some microorganisms, no doubt evolved in entirely different contexts. Another possibility is that of accidental technological transport of microorganisms in the context of space exploration. For instance, it is much easier for Earth-life to be transported to Mars or other planets in the solar system via microbial contamination of unmanned probes, than it is for 
humans, who constructed these probes, to actually expand to Mars, or to other planets, themselves. In fact, despite the extraordinary precautions taken to avoid said contamination (Meltzer 2012), the possibility that viable terrestrial microbes may already be present on Mars, and other solar system locations, cannot be excluded. This possibility highlights the fact that the organisms transported and the ones enabling the transport are not necessarily the same ones. It is also conceivable that specific adaptations for interplanetary transport might evolve in highly expansive IOLCs, although we are in no position to speculate on what those might involve.

\section{Competition between IOLCs}

The vast majority of the universe is extremely inhospitable to life. It is likely that life can only occur on a subset of planets that have certain features that render them habitable, such as a certain range of temperatures, presence of liquid water, or others. Therefore, life is likely to have a very patchy distribution. It may seem problematic for natural selection to act on such widely dispersed entities as IOLCs, because they are likely to come into direct competition very infrequently.

Yet natural selection does not require the selected entities to interact directly or even be close by. For example, Sterelny (1996) argues that selective pressures cannot be equated with physical proximity, concluding that selection can occur at the species level even if the species in question are widely separated, as long as they differ with respect to some character that affects their fitness. He gives the example of widely separated populations infected by the same parasite species (Sterenly 1996). Another example might be selection favoring sexually reproducing species. The species affected by this selective process have been widely separated in space and time, as well as being taxonomically diverse (Okasha 2006). In fact, being able to colonize new habitats, or even the property of being widely dispersed or fragmented into separate populations, can itself be a fundamental driver of species selection (Williams 1992; Sterelny 1996).

Williams (1992) also suggests two cases of natural selection without direct competition: the case of two individual soil arthropods who spend all their lives separated by hundreds of meters yet can be said to be competing for future representation of their genes in the gene pool, and a corresponding example of selection between two species of trout with different vulnerability to climate change. The trout can, in William's (1992) example, be said to be competing for representation in the biota. In the case of IOLC selection, we could say that the different IOLCs are competing for continued existence in the limited habitable space in the universe. This suggests that, at IOLC level, physical-environmental effects on evolutionary change will be preponderant over direct biotic interactions, in accordance with the "Court Jester" hypothesis (Barnosky 2001).

On the other hand, it cannot be ruled out that life forms belonging to different IOLCs might come into direct contact. The same planet might come to be inhabited by two distinct life-clades. This could happen either through the occurrence of two or more distinct abiogenesis events on the same planet, or through the expansion of life to an already inhabited planet. The first scenario was anticipated by Doolittle (2019), who suggests that there might have been other, independently-evolved lineages other than the one that includes LUCA (the last universal common ancestor) that 
did not leave descendants. Should those other lineages have resulted from separate abiogenesis events, that would constitute a case of direct IOLC competition.

While there is very good evidence that all extant life on Earth shares a common ancestor, it is conceivable that on a given planet, possibly one where abiogenesis conditions are highly prevalent, several abiogenesis events might take place simultaneously or in a geologically short time, on different parts of the planet. Later, the different IOLCs could come into contact, but, instead of one IOLC simply outcompeting the others, they might go on to form a hybrid biosphere.

Alternatively, suppose that two independent abiogenesis events take place on the same planet, but there is complete biochemical incompatibility between the two kinds of life that originate. In that case, the two IOLCs would not form a hybrid biosphere; the planet would instead sustain two independent biospheres-this is exactly what has been proposed in the hypothesis of a "shadow biosphere" on Earth (Davies et al. 2009; Cleland 2019, 195-216).

The second scenario in which IOLCs might come into direct contact on the same planet is if an IOLC expands to an already-inhabited planet. This would likely lead to direct competition, the results of which would depend as much on the details of biology as on technological sophistication or cognitive ability. This sort of competition would probably develop along the lines of invasion ecology (Mooney and Cleland 2001), but on a much larger scale.

Another interesting hypothetical case to consider is interplanetary expansion of hybrid biospheres, in cases where more than one IOLC is represented in a planet's biosphere. In that case, interplanetary expansion might involve either one of the two IOLCs, or both, much like a lichen can reproduce by producing a propagule of the two symbiotic partners, but the fungal partner can also reproduce by itself.

It might also happen that an IOLC expands to one or more planets, where it establishes new biospheres and, eventually, a subset of organisms from these independent biotas, which belong to the same IOLC, travel back to the original planet, where it comes into direct competition with the extant biota. This would be a case of intra-IOLC competition, which is equivalent to intra-specific competition in the case of species. For comparison, suppose, for instance, that in a certain bird species, only a small percentage of the population migrates; it is possible that these individuals, or their descendants, later return to the original grounds, where they come into competition with the resident population, which did not migrate.

More dramatically, inter-civilizational encounters have been hypothesized to develop along the lines of the "Dark Forest" rule, with civilizations being destroyed as soon as they are discovered, following a game-theoretic logic of preventative elimination of potential threats (Yu 2015; Kovic 2021). On the other hand, fear of even more technologically advanced hostile civilizations might curtail expansionary programs altogether. The degree to which these considerations might be relevant to inter-IOLC competition will depend on the frequency of a sequence of events, from abiogenesis to the development of technology capability that allows for interplanetary expansion, and also on factors such as how frequently technologically advanced civilizations destroy themselves.

Nevertheless, it is likely that most IOLCs do not come into direct contact. This might seem problematic, since Godfrey-Smith's concept of a Darwinian population, even in the minimal sense, does require "a collection of causally connected individual 
things" $(2009,39)$. This causal connection need not be direct interaction, but it is necessary for the individual things "to be located in a common network of causal interaction - with each other, with environmental conditions, or both" (39-40). If, as seems likely, habitable planets are a restricted subset of all planets, then this is a limited resource for which different IOLCs compete, with highly expansive IOLCs acquiring more of this resource, which then becomes unavailable to other IOLCs (except possibly through direct competition).

\section{Natural selection and life in the universe}

It might be countered that, on the scale of IOLCs, natural selection will never be the most important force; instead, luck will be more important, as it generally is in mass extinctions (Benton 2008, 22). Entire biospheres will often be wiped out by random events, regardless of how complex and well adapted they might be. Yet, while it is true that life is vulnerable to such random catastrophic events, this is also the case with organisms. For example, even if natural selection tends to favor a certain phenotype over another, a healthy individual instantiating the more favorable phenotype will still be vulnerable to random catastrophic events. This does not preclude the effectiveness of natural selection in the long run. On the contrary, expansion beyond the native planet would function as a protection against extinction caused by catastrophic events. IOLCs that have expanded beyond their home planet are less likely to be wiped out by a random catastrophic event, which is why they would have an evolutionary advantage against less expansive or non-expansive ones.

If natural selection does operate at the IOLC level, IOLCs with higher dispersal capacity will become more common in the universe than those with lower or no expansion capacity. We should thus hypothesize the history of life in the Universe to go something like this: once conditions in some parts of the universe became suitable for life, some life forms will have emerged; with time, more life forms appear, while others become extinct; later, we should expect that there will be fewer, but more widely dispersed life forms. In other words, over time, more planets will be occupied by fewer IOLCs. This might, but need not necessarily, involve direct competition between independently originated life clades.

Even if natural selection makes interplanetary expansion more common over time, however, life will always face the problem of the limited availability of habitable planets and the immense distances separating them. These limitations may significantly reduce the chances of galaxy-wide expansion, even on the part of IOLCs with high dispersal capacity. Hence, we should expect life in the universe to occur in small clusters, mainly within specific solar systems with multiple habitable planetary bodies. Furthermore, if interplanetary expansion is often actively carried out by cognitively sophisticated species, then game theoretic considerations involving the risk of elimination by hostile civilizations may play a role in the decision whether to engage in expansion or not.

Crucially, conditions for abiogenesis are more stringent than conditions for habitability, so it is reasonable to suppose that the number of habitable planets in the universe far outnumbers those with conditions for abiogenesis (Cockell et al. 2016). While there are virtually no habitable, but uninhabited, habitats on Earth — that is, life is nearly ubiquitous on Earth-it is likely that there are many planets in the universe 
that are lifeless, despite being habitable (Cockell et al. 2016). These planets constitute available habitats for life forms originated elsewhere that can colonize them.

If there are more planets that can support life than planets with conditions for abiogenesis, and if, over time, more expansive IOLCs are likely to become more common in the universe through natural selection, then some habitable planets where abiogenesis is highly unlikely or even impossible may become secondarily colonized by IOLCs originating from neighboring planets. Therefore, even planets where life cannot originate still have a higher than zero probability of becoming inhabited, and the likelihood of their colonization will increase with the number of potentially life-bearing planets in the vicinity. Thus, solar systems with multiple habitable planetary bodies make exceptionally good targets for astrobiology exploration.

\section{The place of life in scientific theory}

In this section, I would like to assess what the theory proposed in this paper means for the place of life in scientific theory, an important question recently raised by Reydon (2021).

In Hermida (2016), I argued that life on Earth is an individual, based on its historical nature, spatio-temporal continuity, monophyly, and material continuity at the cellular level. However, this idea has been interpreted in different ways. For example, Mariscal and Doolittle (2018) agree that Earth-life is an individual but attempt to draw from it the conclusion that life is not a natural kind, and that it therefore makes no sense to attempt to define life, as we would merely be comparing possible candidates with this one thing, Earth-Life, which, somewhat misleadingly, they call "Life."

However, as Reydon (2021) points out, it does not really follow from the fact that life on Earth is an individual that it is the only one of its kind. The contention (Hermida 2016) that "life on Earth" is but one particular instance of a kind that may have (and probably does have) multiple members is perfectly reasonable, unless one believes that life has appeared only once in the whole universe, which does not seem likely considering its vast scale. While Mariscal and Doolittle are quick to deny that viewing the category of life as "illusory" should in any way compromise research in astrobiology or synthetic biology, it is hard to agree with the claim that their view "should liberate these fields from futile theorizing or overly-restrictive Life-centric criteria for success or failure," when criteria for identifying life is precisely what researchers in those fields cannot do without, even though such criteria must, at present, be tentative.

Reydon (2021) ultimately agrees that the pursuit of definitions of life is a pointless endeavour, but for a different reason. He argues that the search for a definition of "life" should be abandoned because "life" "does not feature as a theoretical term in any scientific theory (in the way that 'species,' 'gene,' and so on do)," and so has no role to play in a naturalistic metaphysics. In particular, Reydon claims that there is no scientific theory in which "life," or more adequately, "Earth-life," features as a particular individual:

While Mariscal and Doolittle $(2018,2)$ specify that life on Earth (or, Life) is a historical entity, they do not show that there is a scientific theory in which the theoretical term "life" features and refers to a particular individual. That 
is, they highlight a possible entity in the world and introduce a proper name ("Life") for it, but do not say whether there is a scientific theory that is about this individual and would allow us to explicate what sort of individual Life is. In other words, the claim that life on Earth is an individual metaphysically underdetermines the nature of life, because there is no scientific theory in which "life" features as a term denoting a particular sort of individual. (Reydon 2021, 3403)

On one hand, it does not seem reasonable to expect that a particular individual (Earth-life) should feature in a scientific theory, since scientific theories are not about individuals, but about kinds. This point is made by Hull $(1976,1978)$, who argues that a scientific theory, such as Darwinian evolution, is about classes of things, such as biological species or organisms, which are kinds of things that could arise anywhere in the universe; it is not about particular species such as swan, which is a spatiotemporally restricted individual, although it applies to it insofar as Cygnus olor is a particular instance of a biological species. In the same way, Newton's theory of gravitation is about physical objects in general, not specifically about Mars, although it applies to Mars because Mars is an example of a physical object.

On the other hand, Reydon does seem to bring up an important point, which might be rephrased in the following way. If Earth-life is an individual but, far from being the only one of its kind, is in fact an example of a category of thing, then we might expect this category of thing to play a role in scientific explanation; otherwise, the justification for the claim that there is such a category of thing seems to be lacking, within a naturalistic metaphysics.

But its absence from scientific explanation might simply reflect the current state of biology in the early twenty-first century. Perhaps the claim that there is no scientific theory featuring the category of thing of which life on Earth is an individual instance could be comparable to the fact that planetary science was profoundly underdeveloped prior to the empirical discovery of extra-solar planets, or that the study of galaxies was seriously lacking in the early twentieth-century, when it was debated whether the Milky Way was the only galaxy in the universe, or whether "elliptical nebulae" were in fact other galaxies.

While Reydon is correct that the claim that life on Earth is an individual is a merely local claim, which refers only to life on Earth, it is nonetheless also the case that this claim is made from quite distinct standpoints. While in Hermida (2016) life on Earth is explicitly posited as an individual instance of a larger category, Mariscal and Doolittle (2018) defend a completely opposite approach, considering life on Earth as the only "Life."

Contrasting Earth-life as an individual with species as individuals, Reydon points to the necessity of theoretical context for these sorts of claims:

[t]he case of species showed that the proponents of the species-are-individuals thesis explicitly assumed the context of evolutionary theory, such that the implicit claim was that species are evolutionary individuals. The case of life showed that the main problem for kinds thinking and individuals thinking regarding life is that the required theoretical context is lacking. There is no theory in which "life" features as a theoretical entity, the argument goes, such that there is nothing for naturalistic metaphysics to do. (Reydon 2021, 3404; emphasis in original). 
In this paper, I have proposed a theory of life in the universe that takes, not life simpliciter, but Earth-life, the large clade composed of the all organisms descending from a particular abiogenesis event, to be an individual instance of a larger category of things in the universe, the category of all independent "trees-of-life" in the universe, or independently originated life clades. I have argued that the set of all IOLCs in the universe forms a Darwinian population that can be subject to natural selection. As in the case of species, evolutionary theory provides both the theoretical context for the claim that Earth-life is an individual and the theoretical framework within which to understand what kind of entity it is an instance of.

\section{Conclusion}

I have attempted to develop a biological hypothesis concerning the application of natural selection to life in the universe. Whatever its merits, it is intended as a serious scientific hypothesis; it is an extension of the domain of application of Darwinian evolutionary theory that, although speculative, is no more fanciful than other such extensions, including some made outside of biology, such as Smolin's (1992) hypothesis of natural selection of universes as an explanation for the apparent fine-tuning of several important cosmological constants.

The proper object of study of biology is life, not life on Earth, which is our locally available sample. Despite its status as a so-called "historical" science, biology is, in principle, no more restricted to our particular planet than planetary science was restricted to our particular solar system prior to the discovery of extra-solar planets, despite their existence being far from certain. In fact, were it not for keen speculation on such possibilities, the research that led to their discovery would never have been carried out.

Acknowledgments. I would like to thank Samir Okasha for his invaluable input. Thanks also to Ford Doolittle and to two anonymous reviewers whose comments greatly improved the manuscript. I am also grateful for the many helpful comments on a poster presentation on this subject matter at the EPSA17 in Exeter, UK. This work was supported by the British Society for the Philosophy of Science through a doctoral scholarship.

\section{References}

Abramov, Oleg, and Stephen J. Mojzsis. 2009. "Microbial Habitability of the Hadean Earth During the Late Heavy Bombardment." Nature 459 (7245):419-22.

Barnosky, Anthony D. 2001. "Distinguishing the Effects of the Red Queen and Court Jester on Miocene Mammal Evolution in the Northern Rocky Mountains." Journal of Vertebrate Paleontology 21 (1): 172-85.

Benton, Michael J. 2008. When Life Nearly Died: The Greatest Mass Extinction of all Time. London: Thames \& Hudson.

Bich, Leonardo, and Sara Green. 2018. "Is Defining Life Pointless? Operational Definitions at the Frontiers of Biology." Synthese 195 (9):3919-46.

Chopra, Aditya, and Charles H. Lineweaver. 2016. "The Case for a Gaian Bottleneck: The Biology of Habitability." Astrobiology 16 (1):7-22.

Ćirković, Milan M. 2019. "Space Colonization Remains the Only Long-term Option for Humanity: A Reply to Torres." Futures 105:166-73.

Cleland, Carol E. 2012. "Life Without Definitions." Synthese 185 (1):125-44.

Cleland, Carol E. 2019. The Quest for a Universal Theory of Life: Searching for Life as we Don't Know It. Cambridge: Cambridge University Press. 
Cleland, Carol E., and Christopher F. Chyba. 2002. "Defining 'Life." Origins of Life and Evolution of the Biosphere 32 (4):387-93.

Cockell, Charles S., Tim Bush, Casey Bryce, Susana Direito, Mark Fox-Powell, Jesse Patrick Harrison, Helmut Lammer et al. 2016. "Habitability: A Review." Astrobiology 16 (1):89-117.

Davies, Paul C. W., Steven A. Benner, Carol E. Cleland, Charles H. Lineweaver, Christopher P. McKay, and Felisa Wolfe-Simon. 2009. "Signatures of a Shadow Biosphere." Astrobiology 9 (2):241-49.

Dawkins, Richard. 1983. "Universal Darwinism." In Evolution from Molecules to Men, edited by D. S. Bendall, 403-25. Cambridge: Cambridge University Press.

Doolittle, W. Ford. 2017. "Making the Most of Clade Selection." Philosophy of Science 84 (2):275-95.

Doolittle, W. Ford. 2019. "Making Evolutionary Sense of Gaia." Trends in Ecology \& Evolution 34 (10):889-94.

Furukawa, Yoshihiro, Yoshito Chikaraishi, Naohiko Ohkouchi, Nanako O. Ogawa, Daniel P. Glavin, Jason P. Dworkin, Chiaki Abe et al. 2019. "Extraterrestrial Ribose and Other Sugars in Primitive Meteorites." Proceedings of the National Academy of Sciences 116 (49):24440-5.

Godfrey-Smith, Peter. 2007. "Conditions for Evolution by Natural Selection." The Journal of Philosophy 104 (10):489-516.

Godfrey-Smith, Peter. 2009. Darwinian Populations and Natural Selection. Oxford: Oxford University Press.

Haber, Matthew H., and Andrew Hamilton. 2005. "Coherence, Consistency, and Cohesion: Clade Selection in Okasha and Beyond." Philosophy of Science 72 (5):1026-40.

Hermida, Margarida. 2016. "Life on Earth is an Individual." Theory in Biosciences 135 (1-2):37-44.

Hull, David L. 1976. "Are Species Really Individuals?" Systematic Zoology 25 (2):174-91.

Hull, David L. 1978. "A Matter of Individuality." Philosophy of Science 45 (3):335-60.

Jablonski, David. 2008. "Species Selection: Theory and Data." Annual Review of Ecology, Evolution, and Systematics 39:501-24.

Knoll, Andrew H. 2003. Life on a Young Planet: The First Three Billion Years of Evolution on Earth. Princeton: Princeton University Press.

Knuuttila, Tarja, and Andrea Loettgers. 2017. "What are Definitions of Life Good For? Transdisciplinary and Other Definitions in Astrobiology." Biology \& Philosophy 32 (6):1185-203.

Korzeniewski, Bernard. 2001. "Cybernetic Formulation of the Definition of Life." Journal of Theoretical Biology 209 (3):275-86.

Kovic, Marko. 2021. "Risks of Space Colonization." Futures 126:102638.

Lenton, Timothy M., Sébastien Dutreuil, and Bruno Latour. 2020. "Life on Earth is Hard to Spot." The Anthropocene Review 7 (3):248-72.

Levin, Samuel R., Thomas W. Scott, Helen S. Cooper, and Stuart A. West. 2019. "Darwin's Aliens." International Journal of Astrobiology 18 (1):1-9.

Lewontin, Richard C. 1970. "The Units of Selection." Annual Review of Ecology and Systematics 1 (1):1-18.

Lineweaver, Charles H., and Tamara M. Davis. 2002. "Does the Rapid Appearance of Life on Earth Suggest that Life is Common in the Universe?" Astrobiology 2 (3):293-304.

Lowe, Donald R., and Gary R. Byerly. 2018. "The Terrestrial Record of Late Heavy Bombardment." New Astronomy Reviews 81:39-61.

Machery, Edouard. 2012. "Why I Stopped Worrying About the Definition of Life ... and Why You Should as Well." Synthese 185 (1):145-64.

Mariscal, Carlos, and W. Ford Doolittle. 2018. "Life and Life Only: A Radical Alternative to Life Definitionism." Synthese 197 (7):2975-89.

Mariscal, Carlos, and Leonore Fleming. 2018. "Why We Should Care About Universal Biology." Biological Theory 13 (2):121-30.

Meltzer, Michael. 2012. When Biospheres Collide: A History of NASA's Planetary Protection Programs. Washington DC: Government Printing Office.

Mooney, Harold A., and Elsa E. Cleland. 2001. "The Evolutionary Impact of Invasive Species." Proceedings of the National Academy of Sciences 98 (10):5446-51.

Okasha, Samir. 2003. "Does the Concept of 'Clade Selection' Make Sense?” Philosophy of Science 70 (4): 739-51.

Okasha, Samir. 2006. Evolution and the Levels of Selection. Oxford: Oxford University Press.

Pross, Addy. 2012. What is life? How Chemistry Becomes Biology. Oxford: Oxford University Press.

Reydon, Thomas A. C. 2021. "On Radical Solutions in the Philosophy of Biology: What Does 'Individuals Thinking' Actually Solve?” Synthese 198 (4):3389-411. 
Ruiz-Mirazo, Kepa, Juli Peretó, and Alvaro Moreno. 2004. “A Universal Definition of Life: Autonomy and Open-ended Evolution." Origins of Life and Evolution of the Biosphere 34 (3):323-46.

Schrödinger, Erwin. 1992. What is life? With mind and matter and autobiographical sketches. Cambridge: Cambridge University Press.

Schulze-Makuch, Dirk, and Louis N. Irwin. 2006. Life in the Universe: Expectations and Constraints. Berlin and Heidelberg: Springer-Verlag.

Segura, Antígona, Sandra I. R. Jiménez, and Irma Lozada-Chávez. 2020. "What is Astrobiology?" In Astrobiology and Cuatro Ciénegas Basin as an Analog of Early Earth, edited by Valeria Souza, Antígona Segura, and Jamie S. Foster, 1-30. Cham, Switzerland: Springer.

Simpson, Carl. 2011. "How Many Levels Are There? How Insights from Evolutionary Transitions in Individuality Help Measure the Hierarchical Complexity of Life." In The Major Transitions in Evolution Revisited, edited by Brett Calcott and Kim Sterelny, 199-225. Cambridge, MA: MIT Press.

Smolin, Lee. 1992. "Did the Universe Evolve?" Classical and Quantum Gravity 9 (1):173-91.

Sterelny, Kim. 1996. "Explanatory Pluralism in Evolutionary Biology." Biology and Philosophy 11 (2): 193-214.

Sterelny, Kim, and Paul E. Griffiths. 1999. Sex and Death: An Introduction to Philosophy of Biology. Chicago: University of Chicago Press.

Van Valen, Leigh M. 1976. "Energy and Evolution." Evolutionary Theory 1 (7):179-229.

Williams, George C. 1992. Natural Selection: Domains, Levels, and Challenges. Oxford: Oxford University Pres. Yu, Chao. 2015. "The Dark Forest Rule: One Solution to the Fermi Paradox." Journal of the British Interplanetary Society 68 (5-6):142-44.

Cite this article: Hermida, Margarida. 2022. "Natural Selection of Independently Originated Life Clades." Philosophy of Science 89 (3):454-470. https://doi.org/10.1017/psa.2021.41 